Transcontinentales

continentales

Sociétés, idéologies, système mondial

5 | 2007

Le défi sanitaire

\title{
La santé entre soins, État et marché
}

Jean-Luc Racine

\section{(2) OpenEdition}

Journals

Édition électronique

URL : http://journals.openedition.org/transcontinentales/681

DOI : 10.4000/transcontinentales.681

ISBN : 978-2-7351-1563-1

ISSN : 1775-397X

Éditeur

Editions de la maison des sciences de l'homme

Édition imprimée

Date de publication : 31 décembre 2007

Pagination : 5-18

ISBN : 978-2-200-92397-6

ISSN : $1950-1684$

Référence électronique

Jean-Luc Racine, "La santé entre soins, État et marché », Transcontinentales [En ligne], 5 | 2007,

document 1, mis en ligne le 21 avril 2011, consulté le 22 septembre 2020. URL : http://

journals.openedition.org/transcontinentales/681 ; DOI : https://doi.org/10.4000/transcontinentales. 681

Tous droits réservés 


\section{La santé entre soins, État et marché}

Le rapport annuel de l'Organisation mondiale de la santé (OMS), publié en 2000, a établi pour l'année 1997 un classement des performances de ses cent quatrevingt-onze États membres en termes d'efficacité du système de santé ${ }^{1}$. La France arrivait alors en tête devant l'Italie et quatre micro-États (Andorre, Malte, San Marin, Singapour), suivis de l'Espagne, d'Oman, de l'Autriche et du Japon. Les États-Unis se classaient $37^{\text {es }}$, à peine deux rangs de mieux que Cuba. Sans surprise, on trouvait en queue de liste une quinzaine de pays africains, et la Birmanie. Dix ans plus tard, la France voit assurément d'un autre œil son système de santé, pour deux raisons.

D'une part, couve le sentiment que le principe de protection maximale au moindre coût permis par un service public de qualité et par l'étendue de la couverture sociale est désormais attaqué. Les décideurs invoquent tout à la fois la nécessité de combler le déficit de la sécurité sociale, l'irresponsabilité d'une part des patients et des médecins conduisant à la surconsommation des médicaments, et les effets du vieillissement de la population qui, affectant les retraites, limiteront aussi l'accès gratuit aux soins. Dans les pays riches où la part de soins assurée (ou remboursée) par l'État s'amenuise sensiblement au nom de l'appel à la responsabilité individuelle, la marginalisation d'une partie de la population n'ayant que peu d'accès aux soins devient un vrai problème de santé publique. L'élection américaine de 2008 se jouera pour partie sur l'Irak, mais aussi sur l'essoufflement délibéré de la protection assurée par Medicare, le système public d'assurance santé, qui n'a d'ailleurs jamais touché qu'une partie des Américains.

D'autre part, le choc du sida, défini comme tel au début des années 1980, a modifié en partie le rapport à la science et la croyance dans les capacités de progrès de la recherche médicale. Pandémie: le mot, qui ne s'applique pas au cancer, est de nouveau d'actualité. Il exprime à sa façon une autre facette de la mondialisation, celle du défi sanitaire. Un sentiment diffus d'insécurité s'est développé, médias aidant, par le biais d'épisodes multiples. D'une part, l'émergence de maladies nouvelles a marqué les esprits à travers le monde: celle la vache folle en GrandeBretagne, la grippe aviaire en Indonésie, le SRAS en Chine, défini par l'Institut

1 - Organisation mondiale de la santé, Rapport sur la santé dans le monde, 2000 : pour un système de santé plus performant, Annexes statistiques, tableau 1, p. 174-177. 
Pasteur comme étant "la première maladie grave et transmissible à émerger au $\mathrm{XXI}^{\mathrm{e}}$ siècle». D'autres part, de nouvelles pathologies ont pris de l'ampleur dans les pays riches qui souffrent de pratiques alimentaires malsaines. Le marché profitable du fast food a vu prospérer un autre marché, épris de produits allégés, d'agriculture biologique, mais aussi de ces "aliments de santé» que sont supposés être les «alicaments» imaginés par l'industrie agro-alimentaire. La malnutrition affectait traditionnellement les pays pauvres. On lui découvre aujourd'hui un sens nouveau, ou le «trop" pèche en symétrie du «trop peu». La menace du bioterrorisme et les effets à moyen terme des dérèglements environnementaux liés au réchauffement climatique ne font qu'ajouter aux incertitudes.

\section{La dichotomie Nord-Sud et I'OMS}

Cet état de fait ne doit pourtant pas occulter la réalité de la question sanitaire mondiale, et la grande dichotomie Nord-Sud qui continue de la régir, pour trois raisons : a) la pauvreté de milliards d'hommes est la cause première de leur mauvaise santé ou de leur incapacité à se soigner convenablement; $b$ ) la puissance publique est inégalement efficace pour mettre en œuvre une politique de santé partout effective; $c$ ) la recherche médicale, celle des multinationales du médicament en particulier, est totalement biaisée en faveur des pays riches, rentabilité oblige. C'est ce que l'Organisation mondiale de la santé appelle depuis quelques années le ratio 90/10 : 90\% de la recherche médicale porte sur des maladies qui affectent $10 \%$ de la population mondiale, celle qui peut payer. Médecins sans frontières le rappela avec force en 2002: sur les 1393 nouveaux médicaments enregistrés dans le monde entre 1975 et 1999, seuls 13 - treize : moins de un pour cent! - étaient consacrés aux maladies tropicales ${ }^{2}$. Si l'espérance de vie s'allonge pour la plus grande part des hommes, elle s'est notablement raccourcie pour un milliard d'entre eux, entre 1990 et 2000.

L'objectif un temps affiché par les Nations unies, "la santé pour tous en l'an 2000 ", n'a donc été qu'un slogan creux. Les objectifs du Millénaire pour le développement ont pris, en cette même année 2000, le relais pour 2015. Certes, l'objectif affiché est celui du développement, mais la santé y joue un rôle essentiel, trois objectifs sur huit y étant explicitement rattachés : ceux qui touchent à la mortalité infantile, à la santé maternelle, au VIH/sida et au paludisme.

Dans son rapport sur la santé et les objectifs du Millénaire, publié en 2005, l'OMS relève quelques avancées repérables sur dix ou quinze ans, telle l'amélioration de la situation nutritionnelle dans une part de l'Asie et en Amérique latine, mais elle constate aussi des reculs, au moins en chiffres bruts, en Asie du Sud et en Afrique, pour souligner au total «la lenteur des améliorations dans le domaine de la santé». La faiblesse des systèmes de santé dans de nombreux pays est aggravée par leur manque d'équité, et par l'anémie des moyens financiers qui leur sont affectés : «il

2 - Clare Kapp, «Policy and people. Bruntland sets out priorities at Annual World Health Assembly», The Lancet, 18 mai 2002. 


\section{LeS OBJeCtifS du MilléNAIRE POUR LE dÉVELOPPEMENT}

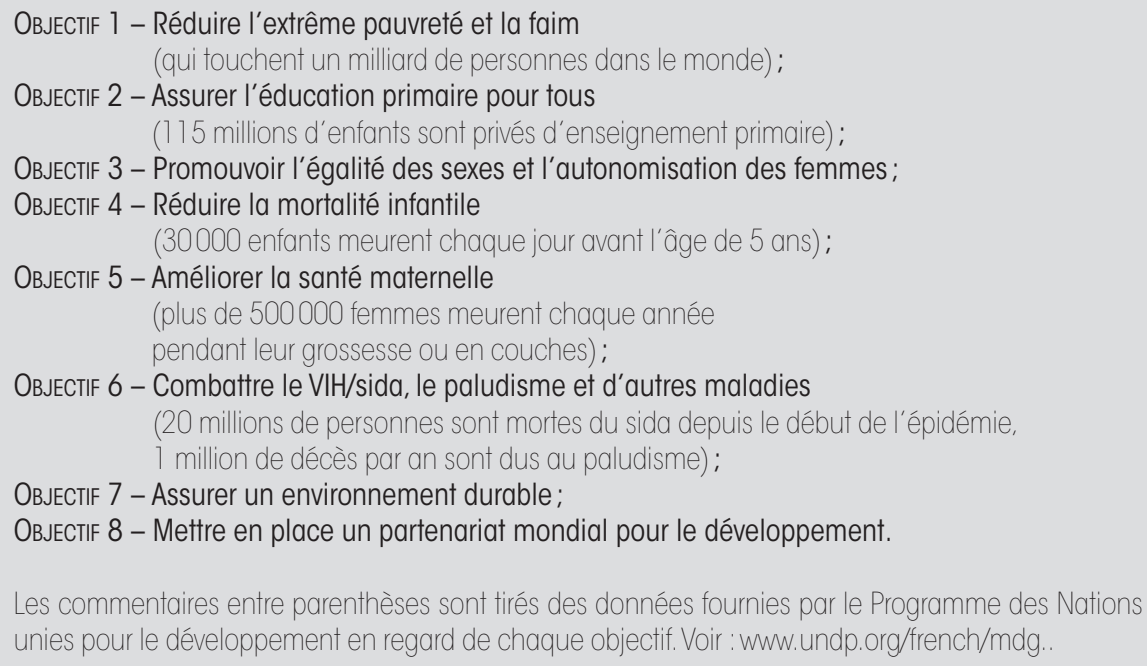

existe un énorme fossé entre les ressources nécessaires et les ressources effectivement disponibles ", constate l'OMS.

Les solutions proposées pour se rapprocher des objectifs affichés paraissent nourries au coin du bon sens. Point n'est besoin d'être grand clerc pour définir les «cinq tâches difficiles" mentionnées dans l'introduction du rapport comme étant les conditions du succès. Elles sont, à vrai dire, d'une banalité affligeante, alors même que la campagne pour les objectifs du Millénaire, engagée en principe dans tous les pays, est d'une ampleur sans précédent ${ }^{3}$. Certes, le corps du rapport est plus informatif et plus programmatique. Il évalue utilement un certain nombre d'opérations, tel le programme DOTS qui, dans plus de cent cinquante pays, accentue le dépistage et le traitement de la tuberculose. Pour cette "grande tueuse», le taux de dépistage mondial a augmenté de $28 \%$ de 2000 à 2003, mais il reste encore très au-dessous de l'objectif, mesuré, de $70 \%$ de dépistage 4 . Ce même rapport rend compte des difficultés d'accès aux médicaments essentiels ${ }^{5}$, et souligne à juste titre le poids du contexte économique dans les taux de morbidité, dont un quart est dû

3 - Les cinq axes stratégiques affichés sont les suivants: a) renforcer les systèmes de santé; b) veiller à ce que l'on accorde à la santé un degré de priorité élevé dans les politiques économiques et de développement général; $c$ ) élaborer des stratégies de santé qui répondent aux besoins divers et changeants des pays; $d$ ) mobiliser davantage de ressources pour la santé dans les pays pauvres; e) améliorer la quantité et la qualité des données sanitaires pour éclairer la prise de décision et favoriser la transparence aux niveaux international et national. OMS, La santé et les objectifs du Millénaire pour le développement, Rapport publié en 2005, p. 8.

4 - Rapport cité, p. 25. DOTS est l'acronyme de Directly Observed Treatment Short course : traitement de brève durée sous observation directe.

5 - Exemple de la lutte contre le sida : "le nombre de personnes recevant des antirétroviraux est passé de 400000 au début de 2004 à près d'un million à la mi-2005. Mais cela ne correspond qu'à une couverture de $15 \%$ parmi les 6,5 millions de personnes, dont les trois quarts vivent en Afrique subsaharienne, qui ont besoin de ce type de traitement» (rapport cité, p. 29). 
aux facteurs environnementaux, en particulier l'insalubrité de l'eau. Il éclaire également la corrélation entre facteurs sociaux et mortalité : une étude réalisée en Inde, souligne le rapport, «semble indiquer qu'une réduction de $10 \%$ du taux d'analphabétisme féminin permettrait de réduire la mortalité infantile de 12,5 pour $1000^{6}{ }^{\prime}$. Au total, ce rapport de l'OMS fournit un diagnostic précieux sur l'état sanitaire de la planète, mais il n'offre guère de perspectives fortes $\mathrm{d}$ 'intervention, et pour cause : au-delà des marges de manœuvre du système onusien, ce sont bien les États qui doivent passer à l'acte, et l'on peut douter que l'invocation du partenariat publicprivé, chaudement recommandé, suffise à faire changer les choses très vite, et dans le bon sens.

\section{La campagne}

\section{pour les médicaments essentiels}

Plus critiques des rapports de force qui conditionnent l'état sanitaire du monde, de grandes organisations non gouvernementales telles que Oxfam International et Médecins sans frontières (MSF) prennent moins de précautions pour dénoncer les logiques financières qui sont en jeu derrière les lacunes de la recherche et les difficultés d'accès aux médicaments. Certes, la science n'a pas réponse à tout. Des maladies que l'on croyait disparues, comme la leishmaniose ${ }^{7}$, réapparaissent sous des formes nouvelles résistant aux médicaments, tandis que de nouvelles maladies se font jour, sans qu'on ait trouvé contre elles vaccins ou traitements. Mais il est aussi des réalités plus troublantes: le constat que les recherches sur les maladies tropicales ont été réduites après la fin de l'ère coloniale, ou le prix de médicaments rendu inaccessible quand est de règle le seul jeu du marché. Exemple, le prix du Fluconazole, un anti-fongique utilisé chez les patients atteints du sida, oscille de 20 dollars par jour pour le médicament breveté au Kenya à 0,60 dollar par jour pour le médicament générique en Thaïlande... Sans parler des médicaments utiles mais abandonnés faute de rentabilité, tel l'Eflornithine, seul médicament efficace contre la maladie du sommeil chez les patients résistant aux traitements classiques, dont la production a cessé en 1995. Dans sa campagne pour l'accès aux médicaments essentiels, MSF peut tout à la fois dénoncer le sort fait aux «malades abandonnés par la science ${ }^{8}$ " en raison du "déséquilibre fatal dans la recherche», et la loi du marché qui hausse exagérément le prix des médicaments, ou qui conduit à déposer des brevets pour des produits en réalité non innovants. Aussi MSF a-t-elle lancé une campagne internationale pour l'accès aux médicaments essentiels. Aux multinationales de la pharmacie qui invoquent la nécessité de faire des bénéfices pour financer la recherche, et qui accusent les médicaments génériques de contribuer aux difficultés des grandes firmes comme Pfizer (48 milliards

\footnotetext{
6 - Rapport cité, p. 45.

7 - Appelée parfois «kala azar»: transmise par un insecte, elle affecte la peau, ulcérée, voire les viscères.

8 - Selon Médecins sans frontières, au cours des trente dernières années, seulement $1 \%$ des médicaments mis sur le marché concernait des maladies comme la tuberculose, le paludisme ou encore la maladie du sommeil. La tuberculose tue 2 millions de personnes par an dans le monde; le paludisme en affecte 500000.
} 
de dollars de vente annuelle) ${ }^{9}$, MSF réplique que «le jeu de la concurrence induite par les médicaments génériques ne menace pas le marché du médicament», passé de 300 à 600 milliards de dollars entre 1999 et 2005, et que dans «un marché en pleine santé", on peut trouver tout à la fois "de plus en plus de brevets, de moins en moins d'innovation " ${ }^{10}$. Oxfam, en parallèle, dénonce "la santé mise à prix» dans son rapport de 2006 intitulé «Des brevets contre les patients ${ }^{11}$ ».

\section{Recherche, profit, et droit à la santé}

Ce constat est au cœur du procès perdu par la firme suisse Novartis, qui a attaqué le gouvernement indien en justice, pour non-respect supposé des normes sur les brevets. En fait, le Parlement indien a voté en 2005 une loi visant à la conformité de la législation nationale avec les principes de l'Organisation mondiale du commerce (OMC). Elle accorde donc des brevets de vingt ans sur les produits véritablement nouveaux. Mais la loi permet aux autorités de récuser un brevet quand il leur apparaît que la transformation d'un produit existant n'apporte pas de progrès sensible en matière de traitement. Une pratique courante des grandes firmes pour étendre les brevets finissants consiste en effet à apporter au produit breveté une modification mineure, afin qu'il soit considéré comme un "nouveau» produit. En 2006, le gouvernement indien a ainsi refusé la demande de brevet déposée par Novartis India pour un médicament anticancéreux, nommé Glivec, à base de mésylate d'imanitib. Novartis est allé en justice, mais a été débouté par la Haute Cour de Madras en août $2007^{12}$. Médecins sans frontières s'en est réjouie, en rappelant que l'Inde, en raison de ses capacités à produire en masse des génériques, est "une source vitale de médicaments à prix abordable pour les pays pauvres». "Principale "pharmacie" des pays pauvres", elle fournit 50 \% des médicaments utilisés dans les pays en développement pour traiter plus de 1,5 millions de malades du VIH/sida (et $80 \%$ des traitements dispensés par MSF dans ses programmes de lutte contre le sida). Le débat public qui s'est ouvert à la suite du jugement de la Haute Cour de Madras illustre parfaitement l'argumentaire des deux parties. Novartis India considère que la Cour, par ce jugement, a signalé qu'elle «ne reconnaît pas l'innovation». Elle met donc en péril, en définitive, "les patients et la santé publique», en refusant de créditer ce que la profession appelle «l'innovation incrémentielle» qui a pourtant fait naître "plus de 70 \% des médicaments aujourd'hui sur le marché». Lui répond le responsable d'une publication spécialisée, Chronicle Pharmabiz, qui souligne trois points. En premier lieu, argumente-t-il, l'innovation invoquée est

9 - Voir le dossier «Billion dollar pills», The Economist, 27 janvier 2007, p. 61-63.

10 - Citations et données de ce paragraphe sont tirées du site Internet de Médecins sans frontières : dossier "Campagne pour l'accès aux médicaments essentiels", dossier sur le procès Novartis contre la loi indienne sur les brevets, et dossier «Recherche médicale» (www.msf.fr, consulté le 29 octobre 2007)

11 - Oxfam International, «Des brevets contre les patients. Cinq ans après la déclaration de Doha", http://www.oxfam.org/fr/policy/briefingpapers/bp95_patentsvspatients_061114 (rapport publié en novembre 2006).

12 - Dans son dossier Novartis, MSF précise que le traitement au Glivec (Gleevec aux États-Unis) revient à 2600 dollars par patient et par mois pour le produit breveté, le générique indien revenant à moins de 200 dollars par mois (www.msf.fr/site/site.nsf/pages/novartis3). 
souvent douteuse. Entre 1978 et 2003, la Food and Drug Administration américaine s'est penchée sur 487 médicaments apparaissant sur le marché. Elle a conclu que «78\% de ces 487 médicaments n'étaient pas meilleurs que les médicaments existants, et que $68 \%$ n'étaient pas vraiment des médicaments nouveaux, mais de vieux médicaments sous des formes et des combinaisons nouvelles ». En deuxième lieu, les multinationales de la pharmacie jouent au maximum la prolongation de leurs brevets, faute de trouver suffisamment de médicaments vraiment nouveaux. L'enjeu financier est énorme, si le brevet est perdu : les ventes annuelles de Lipitor, le médicament anti-cholestérol phare de Pfizer, se montent à 13 milliards de dollars. En troisième lieu, l'étude des quinze majors de la pharmacie laisse penser que leur budget marketing serait deux fois plus gros que leur budget recherche et développement ${ }^{13}$. Le débat sur le juste équilibre entre les droits de propriété intellectuelle et le droit aux médicaments essentiels est loin d'être clos.

Le débat sur l'éthique de la santé se pose dans tous les contextes socio-médicaux ${ }^{14}$. En pays pauvres, il ne se limite pas à la question de l'accès aux médicaments, car il est aussi d'autres dérives, qu'il faut évoquer rapidement. Au rang des controverses, celle qui porte sur les essais clandestins opérés par des grandes firmes sur des patients du Tiers Monde reste ouverte. Le gouvernement du Nigeria s'est ainsi retourné en 2006 contre le laboratoire Pfizer, accusé d'avoir conduit en 1996, sans autorisation, des essais cliniques du Trovan, un médicament contre la méningite, sur plus d'une centaine d'enfants de l'hôpital de Kano. Pfizer se défend, en soulignant que si quelques enfants sont morts, sans que la cause du décès soit avérée, $94 \%$ ont guéri - mais n'évoque guère les contre-effets d'un médicament qui ne fut jamais autorisé en Europe et qui fut interdit aux enfants aux États-Unis ${ }^{15}$. Débouté en octobre 2007, le gouvernement nigérian devrait revenir à la charge. Quelle que soit la vérité sur cette affaire, elle n'est qu'un écho de débats beaucoup plus larges. D'autres essais comparatifs entre placebo et médicament non encore homologué ont été massivement pratiqués en Afrique avec l'azidothymidine ou AZT, un antirétroviral ${ }^{16}$. Et il existe désormais des entreprises, à forte rentabilité, pour opérer au bénéfice des grands laboratoires des essais cliniques dans des pays du Sud, moins regardants sur les règles et moins coûteux que ne le sont les pays avancés ${ }^{17}$.

13 - Voir dans le grand quotidien de Madras, The Hindu, le dossier «Madras High Court : the Novartis judgement», publié le 21 août 2007, avec les contributions opposées de Ranjit Shahani, directeur général de Novartis India : "Choosing innovation benefits India», et de P.A. Francis, rédacteur en chef de Chronicle Pharmabiz: «Patents : a questionable right». Sur le Web: www. thehindubusinessline.com/2007/08/21/stories/2007082151030900.htm et www.thehindubusinessline.com/2007/08/21/stories/2007082151040900.htm.

14 - Voir le dossier de la revue Esprit : «La santé, question de justice », juillet 2007.

15 - Philippe Bernard, "Morts sous antibiotique», Le Monde, 21 juillet 2007.

16 - Voir Yves Mamou, "Éthique et expérimentations médicales», Le Monde, 22 juillet 2007 et l'ouvrage collectif dirigé par François et Emmanuel Hirsch, Éthique de la recherche et des soins dans les pays en développement, Paris, Vuibert, 2005.

17 - Voir Sonia Shah, Cobayes humains, le grand secret des essais pharmaceutiques, Paris, Demopolis, 2007. L'original publié à New York avait un titre moins racoleur : Body Hunters : Testing New Drugs on the World's Poorest Patients, New York, New Press, 2006. John le Carré, dont le best-seller La Constance du jardinier met en scène les multinationales du médicament en Afrique orientale, a préfacé l'ouvrage. 
Un autre problème prend de l'ampleur : la contrefaçon des médicaments, mise sur pied par des mafias chinoises, mais aussi indiennes. Les réseaux de diffusion sont véritablement mondiaux, particulièrement ceux des fameuses "triades» de Hong Kong ou de Taiwan. La vente par Internet de ces faux médicaments facilite plus encore leur diffusion ${ }^{18}$. Quant au trafic d'organes, il est lui aussi un témoignage des dérives du marché frauduleux de la santé, qui est désormais globalisé, avec en tête le commerce des reins, porté par 65000 transplantations en 2005, un chiffre trop élevé pour que les malades des pays riches puissent tous être traités par les circuits de greffe nationaux. Le marché noir s'organise donc autour de cliniques se tournant vers les intermédiaires des vendeurs de rein chinois, indiens, pakistanais, turcs, égyptiens, brésiliens, qui toucheront quelques centaines de dollars, avant que leur rein ne soit transplanté - non sans risques - sur les malades des grands pays acheteurs : États-Unis, Canada, Australie, Japon, Arabie saoudite et Émirats du Golfe. Le coût d'un rein aux Philippines est de 1500 dollars. Il est de 30000 dollars aux États-Unis...

\section{La santé, bien public mondial?}

Se pose en définitive une question majeure: la santé est-elle un bien public mondial? Un bien qui ne serait pas un donné à préserver et à distribuer équitablement, comme l'eau peut l'être par exemple, mais un objectif, défini comme un droit fondamental pour tous. Une note publiée par le Haut Conseil de la coopération internationale - une instance consultative rattachée aux services du Premier ministre - rappelait en 2001 que la surveillance épidémiologique internationale a longtemps été "la première priorité de la politique internationale en matière de santé publique». Cela ne peut évidemment suffire, d'autant que certains États, on le verra ci-dessous, tardent à reconnaître l'apparition d'épidémies. Face à l'inégalité persistante et souvent croissante entre riches et pauvres, aussi bien entre pays qu'au sein de chaque pays, "la santé a du mal à s'imposer comme un bien public mondial». Une telle reconnaissance impliquerait un effort beaucoup plus lourd pour produire des médicaments (y compris pour les maladies délaissées), pour en assurer l'accessibilité financière, et l'accessibilité pratique dans le cadre de systèmes de santé efficaces. Certes, "pour l'OMS, la santé n'est pas une marchandise comme les autres" rappellent les auteurs de cette note, pour regretter toutefois qu'en ce domaine "aucun mécanisme international ne permet de vérifier que ses recommandations sont effectivement mises en ouvre comme à $\mathrm{l}^{\prime} \mathrm{OMC}{ }^{19}$. Si la déclaration

18 - Exemple, l'enquête du New York Times sur l'usage du diéthylène glycol, un solvant très toxique remplaçant la glycérine, beaucoup plus coûteuse, dans des médicaments contrefaits, voire dans des produits de consommation courante comme le dentifrice: Walt Bogdaneich et Jake Hooker, "The poisoning of chinese trade», New York Times, 6 mai 2007, www.nytimes.com/2007/05/06/world/ americas/06poison.html. En juillet 2006, l'Agence française de sécurité sanitaire des produits de santé (AFSSAPS) a lancé une alerte contre ce produit. Les ingrédients toxiques trouvés dans de nombreux produits chinois, sanitaires ou non, ont nourri aux États-Unis une sérieuse méfiance envers les importations, massives, de produits chinois.

19 - Marie Deugnier et Brendan Garrec, «Les enjeux d'une reconnaissance de la santé comme bien public mondial», Paris, Haut Conseil de la coopération Internationale, 2001 (www.hcci.gouv. $\mathrm{fr} /$ lecture/synthese/bien-public-mondial-sante.html). 
des objectifs du Millénaire en 2000, ou la création du Fonds mondial de lutte contre le sida, la tuberculose et le paludisme, en 2002, témoignent à la fois d'une prise de conscience plus aiguisée, de financements accrus et de tentatives de mettre en place des structures opératoires ${ }^{20}$, on reste pourtant très loin du compte.

C'est dans ce contexte que Transcontinentales offre un dossier éclairant quelquesunes des problématiques significatives du défi sanitaire auquel États et sociétés sont confrontés.

\section{Sida, brevets mondiaux \\ et marges de manœuvre étatiques}

Le premier article, de Samira Guennif, porte sur une question essentielle, évoquée plus haut: celle de la gouvernance globale de la propriété intellectuelle et de ses enjeux en matière de santé publique, et particulièrement des traitements anti-sida. Un accord international, dit ADPIC (pour Aspects des droits de propriété intellectuelle touchant au commerce, TRIPS en anglais), a défini en 1994 la norme reconnue par les États membres de l'OMC dans tous les domaines relevant de la propriété intellectuelle. L'accord fait montre d'une certaine flexibilité. Les objectifs de développement et de santé publique étant reconnus, le calendrier d'application de l'accord est étalé dans le temps : dès 1995 pour les pays avancés, 2005 pour les pays émergents, 2015 pour les pays moins favorisés. En outre, les pays membres disposent d'un droit à l'exception, quand ils jugent qu'un brevet est utilisé de façon abusive et contrarie, par exemple, les mesures de santé publique à prendre en cas "d'urgence nationale». Une clause importante, dite "licence obligatoire», permet aux États membres d'autoriser sous certaines conditions la fabrication d'un médicament qui, ailleurs, reste soumis à brevet. La déclaration ministérielle de Doha a du reste concédé que la protection du brevet, certes «importante pour le développement de nouveaux médicaments ", ne pouvait toujours l'emporter contre les urgences de santé publique. Ce cadre étant posé, et ses paramètres techniques complexes étant évoqués, Samira Guennif passe en revue le cas de trois pays ayant défini une politique d'accessibilité aux médicaments anti-sida : le Brésil, la Thaïlande et l'Inde. Le Brésil fut un pionnier en la matière. Il a toujours cherché à négocier des baisses de prix de la part des fabricants, mais sans hésiter à recourir aux «licences obligatoires» pour garantir son programme d'accès universel aux

20 - La «Facilité internationale d'achat de médicaments», baptisée UNITAID, a ainsi été présentée

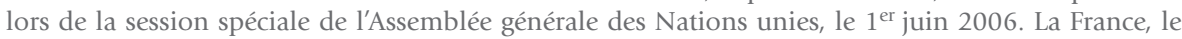
Brésil, le Chili et la Norvège ont joué un rôle majeur dans la conception de ce programme qui cible le sida, la tuberculose et le paludisme, dans les pays en développement, en s'appuyant à la fois sur le système onusien et sur des ONG. Le financement (limité) de ce programme s'appuie sur la "contribution internationale de solidarité sur les billets d'avions" (www.unitaid.eu). À la mi-mars 2007, lors de la conférence de Paris «Couverture du risque maladie dans les pays en développement : rompre le cercle vicieux de la maladie et de la pauvreté», la France a signalé avoir "quasiment multiplié par 30 ses contributions multilatérales dans le secteur de la santé, les portant de 50 millions d'euros en 20002002 à 1,4 milliard d'euros en 2006-2008». La politique française en ce domaine est détaillée sur le site http://www.diplomatie.gouv.fr/fr/actions-france_830/sante_913/index.html. 
antirétroviraux. La Thaïlande, également active face à l'épidémie, illustre combien sont difficiles les négociations, combien sont récurrentes les pressions américaines, et combien la lutte pour des bas prix est toujours recommencée, soit en cherchant à obtenir des concessions des fabricants soit, plus efficacement, en recourrant aux médicaments génériques. L'Inde est un cas plus complexe. On a déjà souligné le rôle essentiel qu'elle joue sur le marché mondial des génériques, et les effets majeurs de sa production pour l'accessibilité des pays pauvres aux soins. Mais l'industrie pharmaceutique indienne - un des secteurs les plus actifs du pays - entend bien dépasser le stade de la copie. Elle se lance aussi dans la recherche de nouveaux médicaments. Le pays adopte donc une "posture sanito-industrielle». Elle limite les critères permettant d'accorder les brevets aux filiales locales des grands groupes pharmaceutiques, en mettant en avant le principe d'efficacité ou de réelle innovation. C'est une façon habile de concilier l'accessibilité financière aux médicaments (encore que le pays soit loin d'avoir une véritable politique d'accès universel aux antirétroviraux) et de respecter les brevets des produits effectivement nouveaux, quitte à négocier des conditions particulières avec les multinationales.

\section{L'Inde à deux vitesses, et le nouveau capitalisme médical}

Avec plus d'un milliard d'habitants, une forte tradition étatique, et une politique de libéralisation mesurée engagée depuis 1991, l'Inde - au-delà du seul cas du sida - offre à l'évidence un exemple particulièrement significatif de réforme des politiques de santé publique. Alors que le pays est un producteur majeur de médicaments génériques et que les biotechnologies y sont un secteur d'avenir, l'Inde n'en reste pas moins au $127^{\mathrm{e}}$ rang mondial quant à l'indice de développement humain, qui combine performances économiques et paramètres sociaux, éducatifs et sanitaires. Alors que le niveau de santé publique reste médiocre (même si l'espérance de vie s'accroît régulièrement), le pays connaît un développement remarqué du secteur hospitalier privé. Depuis une vingtaine d'années, un nouveau capitalisme médical y fleurit, dont le meilleur exemple est celui du groupe Apollo Hospitals, qui se définit comme "le plus important groupe de soins en Asie». Côté en bourse à Bombay, le groupe dispose de 41 établissements de soins, 8000 lits, et plusieurs centres de formation. Il est un des pionniers de ce qu'on appelle par euphémisme le «tourisme médical», en attirant à lui une clientèle étrangère, venue de pays riches pour se faire opérer au meilleur prix. Dans l'étude publiée ici, Bertrand Lefebvre montre que l'essor des soins privés, aujourd'hui majoritaires en Inde, doit moins aux effets d'une politique de réforme libérale qui résulterait d'une posture idéologique, qu'aux échecs de la planification de la santé qui visait, après l'indépendance de l'Inde, à offrir à tous un accès aux soins raisonnable. L'auteur parle ainsi de "privatisation silencieuse des hôpitaux publics indiens" dès avant 1970. Les commissions ayant préconisé d'interdire aux praticiens publics d'accueillir une clientèle privée dans les hôpitaux n'ont pu aboutir, et ont poussé vers le privé une partie du corps médical, d'autant que le nombre de postes publics ne pouvait suffire au nombre de nouveaux diplômés. L'exemple d'un État tel que l'Andhra Pradesh (plus de 60 millions d'habitants) montre aussi comment les classes possédantes rurales ont investi dans l'éducation et dans le secteur médical. En outre, la 
fortune et l'entregent de la diaspora des médecins indiens aux États-Unis a facilité l'investissement dans un nouveau système de cliniques privées de qualité, bien équipées. Investissant trop peu dans le secteur de la santé, l'État est devenu partie prenante d'un système de partenariat public-privé, en phase avec les politiques néolibérales (et non ultra-libérales) des gouvernants, mais dont les résultats en termes d'accès aux soins pour tous sont nettement insuffisants.

\section{L’État défaillant : I’hépatite C en Égypte}

Le cas égyptien pose également la question de l'efficacité des politiques étatiques, mais dans un cadre spécifique de crise : celui de l'épidémie d'hépatite $\mathrm{C}$, affection virale du foie qui frappe l'Égypte de plein fouet, et qui fait d'elle le pays au taux de prévalence le plus élevé pour cette maladie - $13 \%$ selon l'OMS, contre un taux mondial moyen de $3 \%$. Portant une grande attention à la dimension sociale du phénomène, Saadia Radi donne ici les clés multiples d'un dysfonctionnement structurel. Certes, à l'apparition de la maladie, les scientifiques eux-mêmes ont pu hésiter, ce qui allait être reconnu comme l'hépatite C en 1989 étant d'abord baptisé «non A non B». Une fois le mal identifié, les autorités égyptiennes ont tardé à agir, les conséquences de l'épidémie, mal évaluées au départ, leur paraissant «moins gênantes qu'une remise en cause de la politique de santé», d'autant que la demande sociale était faible, à l'inverse de ce qui se passe dans les pays riches où l'opinion publique s'émeut face aux risques sanitaires. En dépit «d'une forte couverture de presse", l'inertie des autorités tient à ce que "la nature autoritaire du régime bloque la protestation sociale», alors même que "la pauvreté des ressources conduit les malades à attendre peu de choses de l'État». Il a fallu attendre, pour mobiliser l'État, qu'une déclaration de médecins égyptiens à l'étranger en 1994 conduise l'Arabie saoudite et des pays du Golfe à exiger des candidats à l'immigration un certificat sanitaire. Les réponses du système de santé égyptien ne furent cependant pas à la hauteur du défi. Quand il apparut qu'une part des contaminés l'avait été par des transfusions sanguines non vérifiées, y compris dans des établissements hospitaliers, le contrôle accru des transfusions ne put suffire, face à la pénurie de sang et aux trafics divers qu'elle suscite. Quant à l'accès aux soins, il est pénalisé, comme souvent, par le prix des médicaments dans un pays sans couverture maladie universelle, et par l'insuffisance de l'accueil dans les hôpitaux publics. Pour des raisons économiques, le système de santé officiel encouragea des soins de substitution, moins chers, mais d'efficacité douteuse. Sans négliger les difficultés proprement médicales à combattre l'hépatite $\mathrm{C}$, l'auteur souligne combien le système de santé détermine largement la qualité de la réponse publique, en amont comme en aval d'une épidémie émergente. Non seulement "le système de santé n'est pas toujours à même de combattre l'épidémie», mais il peut aussi, au pire, être «une niche écologique pour la maladie».

\section{Santé et genre en Russie post-soviétique}

La libéralisation de la Russie post-soviétique offre un exemple très particulier de dégradation des politiques de santé publique. Les soins gratuits pour l'ensemble de la population avaient été un principe majeur de la politique sociale de l'URSS. 
Cet héritage fut remis en cause dans les années 1990, avec des résultats inquiétants qu'exposent Natalia Grigorieva et Tatiana Tchoubarova dans une étude centrée sur la différence des sexes dans le système de santé russe aujourd'hui. D'une façon générale, à la suite de la réduction des investissements d'État, «les principaux indicateurs relatifs à la santé publique se sont détériorés». L'assurance médicale obligatoire "n'a pas permis d'améliorer les moyens médicaux» et la décentralisation du système de santé a eu pour contre-effet d'aggraver les inégalités territoriales. Plusieurs enquêtes statistiques lourdes témoignent d'une hausse générale des taux de mortalité, qui n'est pas seulement due au vieillissement de la population, mais qui traduit avant tout une dégradation des conditions de vie, et les faiblesses des politiques de prévention et de soins. Les auteurs soulignent l'importance, bien trop sous-estimée, des différences de genre dans cette évolution. Les hommes meurent en moyenne beaucoup plus tôt que les femmes, et la hausse différentielle de la mortalité les frappe davantage (+3\%o de 1990 à 2005 à 13,8\%o pour les femmes contre $+6,2 \%$ à $18,8 \%$ pour les hommes). Mais, en sus d'une mortalité croissante, la forte morbidité des femmes s'accentue en raison de la «féminisation de la pauvreté", les femmes russes, qui occupent une forte part des emplois, ayant souvent de bas salaires. Si l'alcoolisme fait des ravages chez les hommes, il s'étend aussi chez les femmes. Quant au sida, il frappe en majorité les femmes, qui restent victimes de préjugés pouvant briser les familles. Natalia Grigorieva et Tatiana Tchoubarova plaident donc pour "analyser en termes de genre les politiques publiques» afin d'améliorer le système de santé et de combattre les discriminations. Certes, les questions de genre restent encore confinées aux cercles d'experts, et n'inspirent que des «mesures disparates" prises au nom de la bonne gouvernance mondiale. Mais elles pourraient à l'avenir devenir "un enjeu politique très concret».

\section{La pluralité médicale : une histoire de l'homéopathie hors d'Europe}

Retour à l'Inde in fine, mais dans une optique différente, celle de la circulation des idées et des pratiques dans un contexte de pluralité médicale. Cette pluralité est constitutive de la société indienne et de son histoire médicale. Bien avant l'introduction de ce que les spécialistes appellent aujourd'hui la biomédecine - le système de référence international issu de la conjonction entre la biologie et la médecine allopathique développée avec l'essor du positivisme scientifique euro-

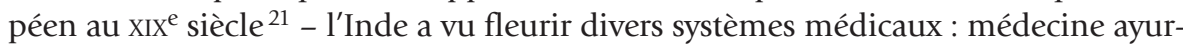
védique, médecine unani, médecine siddha... Ces médecines autochtones n'ont pas été balayées par l'essor de la médecine allopathique. Elles sont aujourd'hui prises en compte par les autorités sanitaires, qui veillent à leur encadrement. Autre médecine allogène au départ, l'homéopathie offre un bon exemple de transfert culturel. Importée en Inde dès les années 1830, diffusée au XIX ${ }^{e}$ siècle par des

21 - «Pour que la biomédecine apparaisse, il a fallu surmonter la prévention des médecins envers la prétention des biologistes à expliquer l'ensemble des mécanismes vitaux, mais également la condescendance des biologistes vis-à-vis de ce qu'ils estimaient être l'incompétence des médecins. » (Jean-François Picard, "Naissance de la biomédecine. Le point de vue d'un historien», Histoire de la médecine et des sciences, $\left.\mathrm{n}^{\circ} 12,1996, \mathrm{p} .97-102.\right)$ 
médecins indiens militants souvent en butte à la Faculté, l'homéopathie a fini par conquérir pleinement droit de cité et reconnaissance officielle, au point que l'Inde est devenue un bastion de l'homéopathie mondiale, et que cette médecine y bénéficie d'une "situation unique de développement", en raison de l'appui public à la recherche. Analysant les étapes de la diffusion de la doctrine, le rôle de l'État dans l'institutionnalisation de l'homéopathie, et les effets de cette institutionnalisation sur les soins et la régulation des pratiques par les organes professionnels, AnneCécile Hoyez et Olivier Schmitz soulignent aussi comment ce succès s'appuie pour partie sur des "ajustements", qui reposent "sur la façon dont les praticiens indiens ont introduit des éléments issus de la culture médicale indienne dans la pratique classique de l'homéopathie». Des études plus avancées sur le cas indien devraient confirmer la capacité de la doctrine homéopathique "à s'implanter dans des contextes culturels très différents, en occupant l'espace laissé vacant par d'autres médecines, pour ensuite s'imposer comme un système thérapeutique complémentaire incontournable».

\section{Entretien :}

\section{pour l'exception sanitaire}

L'entretien avec Rony Brauman, qui clôt ce dossier, permet d'aller plus loin dans l'analyse du défi sanitaire. Aller plus loin, en replaçant dans leur contexte historique les évolutions négatives qu'il faut bien constater. Ainsi du recul de la médecine tropicale après la fin des empires; de la dérive du brevet, inventé pour "protéger l'innovation du pillage des avancées de la recherche», mais devenu aujourd'hui l'instrument d'une «économie de rente» pour les grandes firmes pharmaceutiques qui innovent difficilement; ou des limites de la rhétorique internationale qui lance des slogans tels que "la santé pour tous" sans effets probants, alors même que des champs de coopération effective peuvent être identifiés si l'on prend garde d'asseoir l'intervention médicale et l'accès aux médicaments essentiels sur ce qui permet une véritable politique de santé publique, fondée sur la conjonction entre soins, plateaux techniques supposant investissements d'infrastructure et bonne gestion, et formation médicale continue. Aller plus loin, en soulignant aussi les évolutions positives: l'émergence de l'Asie - Inde, Chine mais aussi Thaïlande - comme nouvelle terre de recherche médicale; l'émergence encore insuffisante mais prometteuse, y compris en Afrique, d'un corps médical plus nombreux, mieux équipé et motivé; la lente prise de conscience de l'impérieuse nécessité, soulignée par Amartya Sen, d'investir dans les clés structurelles du développement que sont l'éducation et la santé. Quant aux enjeux mondiaux d'accès aux soins, un constat paradoxal s'impose : l'Organisation mondiale de la santé, aux objectifs en principe opérationnels, n'est "qu'un immense cabinet conseil», au rôle décisif à cet égard, mais limité faute d'avoir les moyens d'agir directement. À l'inverse, l'Organisation mondiale du commerce, que ses opposants dépeignent comme étant le bastion du libéralisme, s'est plus avancée que l'OMS en reconnaissant qu'en matière de santé, le marché ne peut pas tout, et que des flexibilités sont nécessaires. Pourquoi ce paradoxe? Parce que les pays émergents ont fait entendre leurs voix à l'OMC bien plus qu'à l'OMS, et parce que les mouvements revendicatifs ont beaucoup plus critiqué l'OMC que l'OMS, poussant ainsi l'Organisation du commerce à réguler, 
négociations étatiques et pressions militantes obligent, à consentir des aménagements sensibles au bien public. Un pas de plus, et il conviendrait de reconnaître "l'exception sanitaire», aux enjeux aussi décisifs que «l'exception culturelle». Ce rôle de l'opinion publique et des débats d'idées se manifeste également dans les deux thèmes qui terminent cet entretien : la nouvelle vision élargie du concept de «crise sanitaire» et la montée en puissance du "principe de précaution». Dans les deux cas, les préoccupations sanitaires amènent à ne pas sous-estimer les risques. Encore faut-il ne pas sombrer dans une «théologie de la peur» suscitant une rétraction anxiogène, et faire face aux menaces dans un esprit constructif.

\section{Variations}

\section{Le déni de pauvreté au Japon}

Deux articles complètent ce numéro. Le premier traite de la pauvreté dans un pays riche : le Japon. Mélanie Hours y décrit l'émergence du problème et analyse l'évolution des systèmes de représentation de la pauvreté. Longtemps niée comme phénomène significatif, la pauvreté était le plus souvent dépeinte en termes de marginalité sociale. La récession des années 1990 a mis à mal le modèle établi du travail garanti à vie dans l'entreprise, et le nombre de travailleurs précaires s'est accru. "Main-d'œuvre jetable» (comme dans le secteur de la construction fonctionnant largement avec des journaliers) et "laissés-pour-compte de la société japonaise» restent victimes de l'ostracisme qui les frappe, tout en intériorisant "au moins partiellement le jugement accusateur et le mépris que les Japonais "ordinaires" portent sur eux». Les sans-abri, dont les deux tiers travaillent, sont peu nombreux selon les chiffres officiels, mais un livre blanc leur a toutefois été consacré en 2001, année qui marque une inflexion de la politique publique à l'égard de tous les défavorisés. Le confirme la loi de réinsertion sociale des homeless de 2002 (le terme anglais est utilisé au Japon). Pour autant, «l'assistance publique dans les dépenses totales de sécurité sociale est en baisse continue» et les procédures d'aide sont décourageantes : loi ambiguë, critères draconiens, manque délibéré d'information expliquent que nombre de personnes éligibles ne bénéficient pas d'un droit à l'assistance au total peu efficace. Si «les prémices d'une évolution dans les représentations de la pauvreté» au Japon s'esquissent aujourd'hui, la pratique du déni domine toujours. Le mot même de pauvreté est banni de la presse japonaise, sauf pour l'évoquer dans un contexte historique, ou à propos des pays étrangers. L'évolution modeste de la loi ne s'appuie pas encore sur une véritable prise en compte du problème dans les mentalités collectives. Derrière les subterfuges du vocabulaire, l'imaginaire social dominant continue de définir les diverses situations de précarité comme des «déviations par rapport à la norme sociale».

\section{Paradigmes de la mémoire}

Ce numéro de Transcontinentales paraît peu après la tenue du premier colloque des instituts français de recherche à l'étranger, organisé au musée du quai Branly sur le thème "Présences du passé. Mémoires et sociétés du monde contemporain». 
Nous publions ici la conférence inaugurale du colloque, prononcée par MarieClaire Lavabre, dont le livre Le fil rouge. Sociologie de la mémoire communiste fit date à sa sortie en 1994. Synthétisant ici des années de recherches individuelles et collectives, Marie-Claire Lavabre distingue trois paradigmes dominant l'analyse du phénomène mémoriel. Celui de Pierre Nora, qui dirigea en historien les volumes sur Les lieux de mémoire, "se donne pour objet la généalogie des représentations du passé, les mises en récit publiques du passé et plus encore les usages politiques du passé». C'est le paradigme qui s'est sans doute le plus diffusé, et qui a mis en lumière (parfois trop?) la dichotomie entre histoire et mémoire. Le second paradigme, celui du philosophe Paul Ricœur, met en avant le nécessaire «travail de mémoire», que Marie-Claire Lavabre résume ainsi : «les sociétés, comme les individus, peuvent être malades de leur passé. Elles ont à accomplir un "travail de mémoire" comme on fait un travail de deuil pour atteindre la "juste mémoire", le bon oubli et la réconciliation avec l'autre autant qu'avec soi-même». Beaucoup reste à faire, dans maintes sociétés déchirées, ou dans maintes régions traumatisées, pour en arriver là. Troisième paradigme, le plus ancien, celui du sociologue Maurice Halbwachs qui, le premier en France, souligna la nécessité pour les sciences sociales de s'attacher aux problématiques mémorielles. L'objectif, ici, est essentiellement intellectuel : en invitant à l'analyse des "cadres de la mémoire», Halbwachs propose «d'ouvrir la boîte noire, de penser les interactions entre usage du passé et souvenirs, de vérifier empiriquement ce que sont les représentations partagées du passé et donc de répondre à la question qui devrait se poser en amont : peut-on influencer la mémoire, et à quelles conditions?» Au total, un constat s'impose : la mémoire est devenue envahissante, objet conceptuel et réel à la fois, manifeste dans l'appel au "devoir de mémoire», occulté, mais seulement en apparence, dans les stratégies de déni ou dans les "pactes d'oubli» qui cherchent à cautériser les plaies de la mémoire mais ne le peuvent sans doute qu'au prix des difficiles exercices de «justice et réconciliation». "Pactes d'oubli»? La formule paraît bien incertaine, car un tel pacte suppose non pas l'oubli, mais le dépassement du passé, par un travail en commun dont on sait combien il est difficile, et combien il suppose d'abord la reconnaissance partagée des faits. C'est là où les historiens et les militants de la mémoire travaillent différemment, dans les situations les plus délicates, face aux traumatismes ineffaçables, a fortiori quand les histoires nationales divergent sur des "passés qui ne passent pas» et qu'une partie, contre l'autre, veut voir reconnaître sa vérité, celle des victimes. Arménie et Turquie depuis bientôt un siècle, Chine et Japon depuis plus de soixante ans, et plus près de nous l'Afrique des grands lacs nous rappellent avec insistance, entre bien d'autres cas, dont celui de l'esclavage, ce que saint Augustin avait évoqué comme étant "le présent du passé». "Présences du passé» : c'est précisément le titre donné à ce premier colloque des instituts, qu'a introduit la réflexion ici publiée. 